\title{
3-D Turbulence Numerical Simulation for the Flow Field of Suction Cylinder-Seeder with Socket-Slots
}

\author{
Yanjun $\mathrm{Zuo}^{1}, \mathrm{Xu} \mathrm{Ma}^{1,2, * *}$, Long $\mathrm{Qi}^{1}$, and Xinglong Liao ${ }^{1}$ \\ ${ }^{1}$ College of Engineering, South China Agricultural University, Guangzhou, P.R. China \\ ${ }^{2}$ Key Laboratory of Key Technology on Agricultural Machine and Equipment, \\ Ministry of Education, South China Agricultural University, Guangzhou, P.R. China \\ 42648135@qq.com, maxu1959@scau.edu.cn, qilong@scau.edu.cn, \\ liaoxinglong1985@163.com
}

\begin{abstract}
The flow field has significantly impact on seeding performance in the suction seeding device. A three-dimensional, incompressible, viscous, RNG turbulence model and the SIMPLE method were used by computational fluid dynamics(CFD), and the flow fields of suction cylinder-seeder with different socket's radiuses were simulated by Fluent. When vacuum is $4 \mathrm{kPa}$ and productivity is 350 trays/h, the simulant results show that pressure is uniform, velocity is stable, energy loss mainly occurs near slots and outlet, and there is less interaction among socket-slots; The effect of flow field on socket's radius to the cylinder isn't significant by contrasting different socket's radiuses on the average turbulent kinetic energy, the average vacuum and the maximum difference of velocity behind slots; The experimental results show that the best seeding performance is $84.73 \%$ when the socket's radius is $5.5 \mathrm{~mm}$. Although the performance should be improved, but any sockets are never plugged, which shows enough that the seeder is a very promising precision seeding device.
\end{abstract}

Keywords: Socket-slot, Suction cylinder, Flow field, Numerical simulation.

\section{Introduction}

Usually, the demand of high-precision seeding is $2 \pm 1$ seeds/bowl for super hybrid rice tray nursing seedlings, and can't be satisfied invariably by the traditional mechanical seeding device. The suction seeding device is becoming mainstream for super hybrid rice with its advantage of low broken-seed rate, high single-seed rate, good generality, imprecise demand of seminal dimension and so on[1-2]. Flow field impacts seeding performance significantly in the suction seeding device, so it has been studied by many researchers at home and aboard. In overseas, Karayel D etc. have built mathematical model of vacuum pressure on a precision seeder[3], Guarella P etc. have studied the performance of a vacuum seeder nozzle for vegetable seeds in experiment and

The paper is supported by the National Natural Science Fund Projects (Project number is 50775078), the National "11th Five-Year Plan" to support Projects (Project number is 2006BAD28B01-3), the earmarked fund for Modern Agro-industry Technology Research System and the fund for indraught of person with ability in colleges of Guangdong.

* Corresponding author. 
theory[4]. In domestic, Yuan Yueming, Wang Zhaohui etc. have simulated and experimented the flow field for suction seeders of vertical disc and cylinder[5-6], Li Yaoming etc. have analyzed the flow field of sucking nozzle to suction seeder[7]. Suckers of existing suction seeding devices are usually plugged during seeding because rice is seeded with sprout. Although two-layer suction cylinder-seeder is developed by Pang Changle[8] and could relieve the suckers plugging, it can not satisfied the requirement of continuous seeding. So a seeding device will be developed with a new theory and new structure to solve this problem. Based on the traditional suction cylinders, a suction cylinder-seeder with socket-slots is developed which can solve the problem of suckers plugging effectively through seed-filling, seed-sucking, seedclearing and forcible sucker-clearing.

In order to improve the seeding performance of suction cylinder-seeder with socket-slots, the numerical simulations for different socket's radiuses have been calculated by software Computational Fluid Dynamics (CFD) in this paper. Socket's radius was optimized by analyzing the distributions of velocity and pressure inside the cylinder, and checked by experiment. This study provides theoretical and practical basis for the design of super hybrid rice seed-metering device for nursing seedling.

\section{Principle of Suction Cylinder-Seeder}

The suction cylinder-seeder with socket-slots for experiment is shown in figure 1 . After flowing out of the seed hopper, rice seeds fall onto vibrant board. Then the

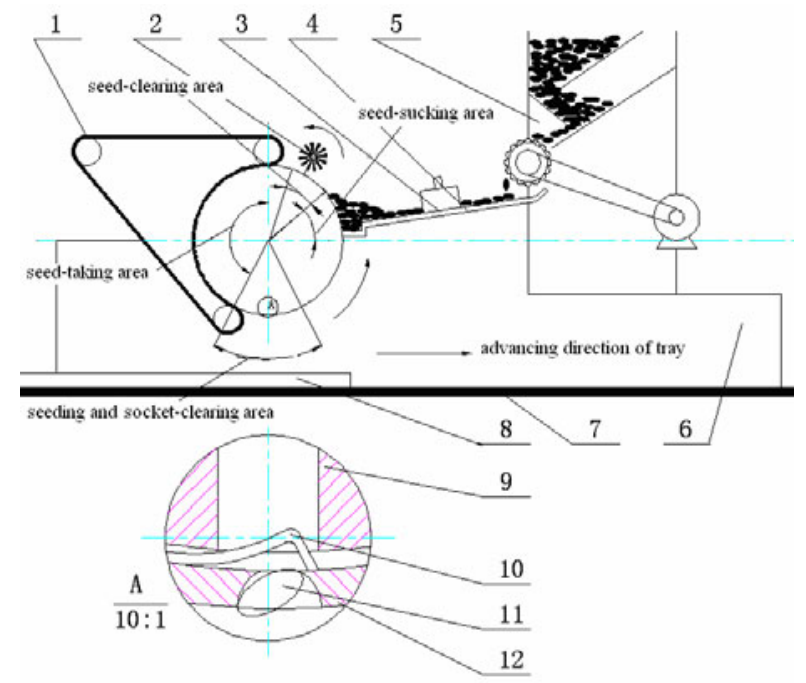

Fig. 1. Principe diagram of suction cylinder-seeder with socket-slots 1 Seed-protecting belt, 2 Seed-clearing rolling brush, 3 Suction vibrator, 4 Vibrant board, 5 Seed hopper, 6 Frame, 7 Conveyor belt, 8 Tray, 9 Pressure chamber, 10 Seed-popping springs, 11 Rice seeds, 12 Cylinder 
seeds worm downward along with vibrant board under vibration and enter seedsucking area where prickle, broken sprout and other impurities are removed through the sieve meshes. The seed-feeding is accomplished under the gravity and vacuum. When the sockets rotate to seed-clearing area, the redundant seeds are cleared firstly by seed-clearing rolling brush rotating at the same direction with cylinder, and the residual seeds in sockets rotate into seed-protecting belt continually. The vacuum will be cut off when the sockets rotate into seeding and socket- clearing area, the springs pop the seeds and clear the sockets forcibly. The seeds fall into the appointed bowl under the gravity and elasticity, so realizing the precision seeding.

\section{Numerical Simulation}

\subsection{Physical Model}

Along the axis, fifteen rows of sockets $(\mathrm{R}=4.5 \mathrm{~mm}, 5.0 \mathrm{~mm}, 5.5 \mathrm{~mm})$ that corresponding to the tray of $15 \times 25$ bowls are made outside cylinder. Along the circumference,

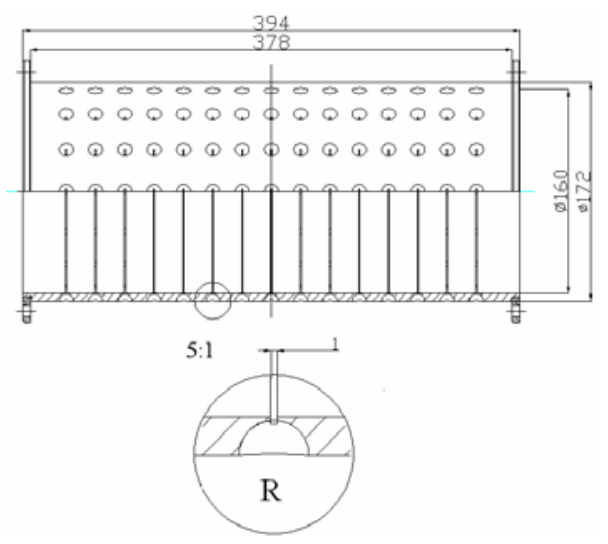

Fig. 2. Structure of cylinder

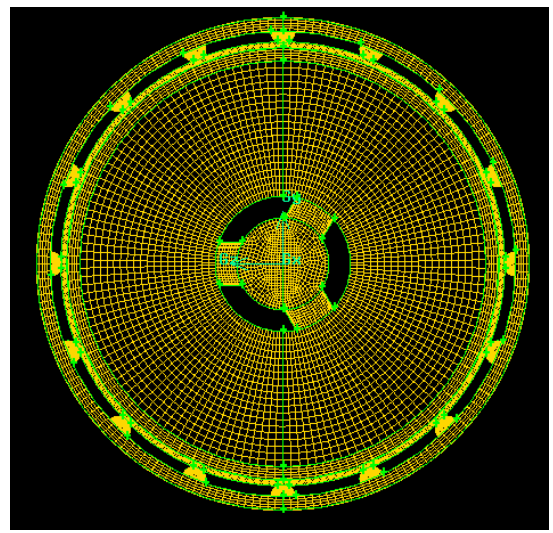

Fig. 3. Computational model of cylinder 
fifteen ring grooves are also made at the corresponding place of the sockets inside cylinder. The sockets and ring grooves are intersecting and forming a slot of $4.0 \mathrm{~mm}$. The specific dimensions are shown in figure 2. Because there are larger differences in local sizes, the model uses structured and unstructured grids to mesh, as is shown in figure 3 .

\subsection{Flow Equations}

Rotation of cylinder, two different flow fields which are connected by socket-slots and the effect of air viscidity, so turbulent swirling flow is formed starting from inlet of socket-slots nearby the insides of cylinder and socket-slots, and contains laminar flow and swirly shear flow at the wall of cylinder, jet flow of slots, free flow in the pipeline, flow with separation and so on. In order to ensure the accuracy of numerical simulation, turbulence model of RNG $k-\varepsilon$ is used in this paper. In the model flow state, spatial coordinates, rotation and swirling flow state in the average flow have been considered, turbulent viscosity has been modified. And the model supplied an analytical formula with low Reynolds number which is more accuracy than standard equation of $k-\varepsilon$ to the flow of near wall[9]. Turbulent kinetic energy and turbulent dissipation rate were calculated as follows:

$$
\begin{gathered}
\frac{\partial}{\partial t}(\rho k)+\frac{\partial}{\partial x_{j}}\left(\rho k u_{i}\right)=\frac{\partial}{\partial x_{j}}\left(\alpha_{k} \mu_{e} \frac{\partial k}{\partial x_{j}}\right)+G_{k}+G_{p}-\rho \varepsilon \\
\frac{\partial}{\partial t}(\rho \varepsilon)+\frac{\partial}{\partial x_{i}}\left(\rho \varepsilon u_{i}\right)=\frac{\partial}{\partial x_{j}}\left(\alpha_{\varepsilon} \mu_{e} \frac{\partial \varepsilon}{\partial x_{j}}\right)+\frac{\varepsilon}{k}\left(C_{1 \varepsilon} G_{k}-C_{2 \varepsilon} \rho \varepsilon\right)
\end{gathered}
$$

Where: $k$ is the turbulent kinetic energy in $\mathrm{m}^{2} / \mathrm{s}^{2} ; \varepsilon$ is the turbulent dissipation rate in $\mathrm{m}^{2} / \mathrm{s}^{3} ; G_{\mathrm{k}}$ is the generation item for turbulent kinetic energy $(k)$ caused by average velocity gradient; $G_{\mathrm{p}}$ is the generation item for turbulent kinetic energy $(k)$ caused by buoyancy; and $\mu_{\mathrm{e}}$ is the turbulent viscosity in $\mathrm{Pa} \cdot \mathrm{s}$, and $\mu_{\mathrm{e}}=\rho C_{\mu} k^{2} / s$.

Model constants[10] are $C_{1 \varepsilon}=1.42, C_{2 \varepsilon}=1.68, C_{\mu}=0.0845, \alpha_{\mathrm{k}}=\alpha_{\varepsilon}=1.39$.

\subsection{Boundary Condition}

The fluid is normal temperature air under standard condition, and its density is $1.205 \mathrm{~kg} / \mathrm{m}^{3}$, viscosity is $1.83 \times 10^{-5} \mathrm{~Pa} \cdot \mathrm{s}$, and temperature is $293 \mathrm{~K}$, so the inlet and standard pressures are all $101325 \mathrm{~Pa}$. The pressure inlet and outlet are all subsonic speed, and the wall uses adiabatic and no-slip boundary conditions[11].

\section{Simulation Results and Analyses}

Numerical simulations for suction cylinder-seeder with three socket's radiuses $(\mathrm{R}=4.5 \mathrm{~mm}, 5.0 \mathrm{~mm}, 5.5 \mathrm{~mm}$ ) use the method of semi-implicit method for pressurelinked equations(SIMPLE) when outlet pressure is $97325 \mathrm{~Pa}$ and productivity is 350 trays $/ h$, and the results are shown in figure 4 and table 1 . 


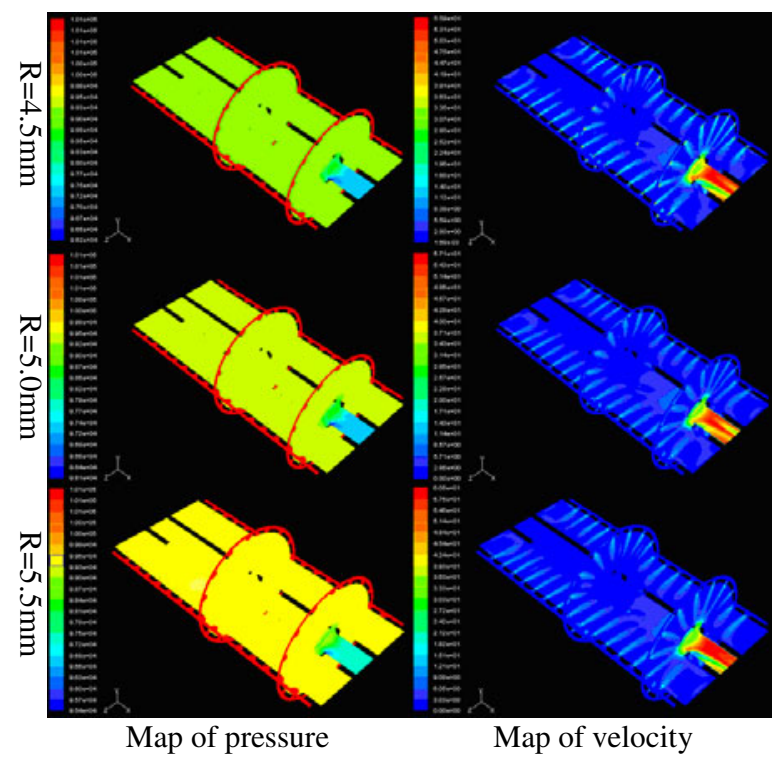

Fig. 4. Distribution map of pressure and velocity

From the map of pressure in the figure 4, it can be known that pressure distributes uniformly in the whole cylinder and changes greatly at the slots and the outlet. The main reason is that, air fluid can't turn suddenly like the wall as the inertial force is dominating when section of pipeline changes suddenly, disengage phenomena of main flow area and wall is occurring, and then swirling area forms. Distributional adjustment of velocity in main flow area, rotation of fluid in the swirling area and exchange of fluid particle in the two areas, all these lose energy, so energy loss occurs near the slots and outlet. From the map of velocity in the figure 4 , it can be known that velocity is stable inside the cylinder, air fluids flow through socket-slots and have less interaction in the axial and circumference.

Table 1. Contrast of different socket's radiuses

\begin{tabular}{llll}
\hline $\mathrm{R}(\mathrm{mm})$ & $k_{a}\left(\mathrm{~m}^{2} / \mathrm{s}^{2}\right)$ & $V_{a}(\mathrm{kPa})$ & $v_{\max }(\mathrm{m} / \mathrm{s})$ \\
\hline 4.5 & 1.7838 & 2.10 & 13.98 \\
5.0 & 1.7892 & 2.04 & 11.47 \\
5.5 & 2.0793 & 1.88 & 9.22 \\
\hline
\end{tabular}

Note: $k_{a}$ is the average turbulent kinetic energy; $V_{a}$ is the average vacuum behind slots; and $v_{\max }$ is the maximum difference of velocity behind slots. 
The contrasts of different socket's radiuses on average turbulent kinetic energy, average vacuum and maximum difference of velocity after slot are shown in table 1 . It can be known that: average turbulent kinetic energy increases along with the increases of socket's radius, because the bigger socket's radius, the stronger turbulization, the more significant effect of flow on eddy current, and the less stable flow field; average vacuum decreases along with the increases of socket's radius, because the bigger socket's radius, the more friction loss when air fluid through socket-slots; maximum difference of velocity decreases along with the increases of socket's radius, because the bigger socket's radius, the longer distance of air fluid pass socket-slots, the smaller difference of velocity, and the more stable seed-sucking; the effect of flow field on socket's radius isn't significant. The amount of seed-feeding is affected by socket's radius, so the seeding performance would be checked.

\section{Conclusions}

\subsection{Experimental Material}

The experimental material is super hybrid rice of Peizataifeng. Its dimension is shown in table 2.

Table 2. Dimensions of gemmative rice seed

\begin{tabular}{ccc}
\hline Length $(\mathrm{mm})$ & $\operatorname{Width}(\mathrm{mm})$ & Thickness $(\mathrm{mm})$ \\
\hline 9.23 & 3.45 & 2.42 \\
\hline
\end{tabular}

\subsection{Experimental Procedures}

1. Rice seeds are soaked and germinated.

2. The suction vibrator provides a stable pressure of $0.2 \mathrm{MPa}$ through fan setting.

3. Vacuum of $4 \mathrm{kPa}$ is got by vacuum pump setting.

4. The productivity of 350 trays $/ h$ is obtained by converter adjusting.

5. The seed-clearing rolling brush starts and rotates at the same direction with cylinder, and its speed of 50rpm.

6. The seeds are put into seed hopper and vibrator starts, the vibrant board begins vibrating. And then the seed hopper starts and begins feeding seeds to the cylinder. Finally, seeding is experimented after the running is stable.

\subsection{Experimental Factor}

According to the result analyses which were simulated by Fluent, control variable method is used to study the effect of seeding performance on socket's radius, and the results of numerical simulation were checked. 


\subsection{Experimental Plan and Results}

The experiment has been done on the test-bed for nursing seedling at College of Engineering, South China Agricultural University. The main index is qualified rate (bowls of $1 \sim 3$ seeds/all bowls $\times 100 \%$ ))to be examined. The results are shown in table 3 .

Table 3. Experimental results

\begin{tabular}{cccc}
\hline $\mathrm{R}(\mathrm{mm})$ & $R_{q}(\%)$ & $R_{r}(\%)$ & $R_{c}(\%)$ \\
\hline 4.5 & 78.89 & 16.47 & 4.64 \\
5.0 & 81.69 & 11.74 & 6.57 \\
5.5 & 84.73 & 6.31 & 8.96 \\
\hline
\end{tabular}

Note: $R_{q}$ is the qualified rate, $R_{r}$ is the reseeding rate, and $R_{c}$ is the cavity rate.

It can be known from table 3 that, when socket's radius is smaller, the seeds get in the sockets uneasier and are removed easier by seed-clearing rolling brush and the cavity rate is higher; when socket's radius is bigger, the amount of seeds enter the sockets is larger and the reseeding rate is higher; the rising extent of reseeding rate is less than the falling extent of cavity rate because of seeding-clearing rolling brush, so the qualified rate increases along with the increases of socket's radius. The seeding performance of suction cylinder-seeder with socket-slots is the highest $(84.73 \%)$ when the socket's radius is $5.5 \mathrm{~mm}$.

\section{Conclusions}

Through the simulation and experiment of different socket's radiuses when the vacuum is $4 \mathrm{kPa}$ and the productivity is 350 trays $/ h$, the conclusions are obtained as follows:

1 The distribution of pressure is uniform, velocity is stable, energy loss occurs mainly near the slots and outlet, and air fluid has less interaction among the slots in the cylinder.

2 The effect of flow field on socket's radius is not significant to the cylinder, and the seeding performance is checked.

3 The experimental results show that the best seeding performance of suction cylinder-seeder is $84.73 \%$ when the socket's radius is $5.5 \mathrm{~mm}$.

In fact, there are many factors affecting the seeding performance, such as vacuum, productivity and positional angle of seed-feeding. Each factor is not the optimum as preliminary study, so the capability is not high at present, and will be studied further. There isn't any sockets that are plugged in the whole process of experiment, which 
shows that the suction cylinder-seeder with socket-slots is a very promising precision seeding device.

\section{Acknowledgements}

The paper is supported by the National Natural Science Fund Projects (Project number is 50775078), the National "11th Five-Year Plan" to support Projects (Project number is 2006BAD28B01-3), the earmarked fund for Modern Agro-industry Technology Research System and the fund for indraught of person with ability in colleges of Guangdong.

\section{References}

1. Zhou, H., Ma, X., Yao, Y.: Research advances and prospects in the seeding technology and equipment for tray nursing seedlings of rice. Transactions of the CSAE 24(4), 301-306 (2008) (in Chinese)

2. Wu, M., Tang, C., Li, M., et al.: The present situation and countermeasures about seeding apparatus of paddy precision seeder. Chinese agricultural mechanization 3, 30-31 (2003) (in Chinese)

3. Karayel, D., Barut, Z.B., Ozmerzi, A.: Mathematical Modelling of Vacuum Pressure on a Precision Seeder. Biosystems Engineering 87(4), 437-444 (2004)

4. Guarella, P., Pellerano, A., Pascuzzi, S.: Experimental and Theoretical Performance of a Vacuum Seeder Nozzle for Vegetable Seeds. Journal of Agricultural Engineering Research (64), 29-36 (1996)

5. Yuan, Y., Ma, X., Jin, H., et al.: Study on vacuum chamber fluid field of air suction seedmetering device for rice bud-sowing. Transactions of the CSAM 36(6), 42-44 (2005) (in Chinese)

6. Wang, Z., Ma, X., Dong, R., et al.: Numerical simulation for air field of air-suction cylinder seeder. Journal of Jilin Agricultural University 31(6), 781-784 (2009) (in Chinese)

7. Chen, J., Li, Y., Wang, X., et al.: Finite element analysis for the sucking nozzle air field of air-suction seeder. Transactions of the CSAM 38(9), 59-62 (2007) (in Chinese)

8. Changle, P., Zhuomao, E., Su, C., et al.: Design and experimental study on air-suction towlayer cylinder rice seeder. Transactions of the CSAE 5(9), 52-55 (2000)

9. Wang, F.: Analysis of Computational Fluid Dynamics: Theory and Application of Software CFD, pp. 124-125. Tsinghua University Press, Beijing (2005) (in Chinese)

10. Wu, B., Yan, H., Zhang, J.: Study on 3-D turbulent numerical simulation and performance foreacast of slurry pump. China Mechanical Engineering 20(5), 585-589 (2009) (in Chinese)

11. Deng, D.: Fluid flow handbook, p. 471. China Petrochemical Press, Beijing (2004) (in Chinese) 